\title{
The differential diagnosis of 'little strokes'
}

\author{
JohN MARShaLL \\ M.D., F.R.C.P.(Ed.), F.R.C.P.(Lond.), D.P.M. \\ Reader in Clinical Neurology, University of London \\ National Hospital for Nervous Diseases, Queen Square, London, W.C. 1
}

\section{Summary}

Transient focal disturbances of cerebral function are often indicative of atherosclerosis. They may occur, however, in association with a number of other conditions which are illustrated by fourteen brief case histories.

\section{Introduction}

The more widespread appreciation of the fact that transient ischaemic cerebrovascular attacks (TIAs) often give warning of an impending completed stroke has been a significant advance in the management of cerebrovascular disease. An increasing number of patients are now referred for investigation after they have experienced only one or two TIAs while there may still be time to prevent the development of a completed stroke.

Whilst the pathological basis for the great majority of TIAs is atheroma, it should not be forgotten that they may be associated with other types of pathology. Similarly, focal neurological disturbances which are too prolonged to justify the label TIA, and yet, by remitting, do not earn the title of a completed stroke, may also be associated with a pathology other than atheroma. The purpose of the present paper is to call attention to some of these by recounting brief case histories of fourteen patients who were encountered in a series of 900 personal cases of cerebrovascular disease.

\section{TIA and cardiac dysrhythmia}

The occurrence of TIAs in association with episodic cardiac dysrhythmia is illustrated by the two following cases.

Case No. 778

A man of 41 years gave a 5-year history of sudden attacks of unsteadiness of gait or stance, each lasting about $1 \mathrm{~min}$ and recurring at monthly intervals. They were not associated with vertigo, visual symptoms or feelings of faintness, but he was aware on each occasion of a fast, regular beating of his heart.

Neurological examination was negative, the heart was normal, and the blood pressure 150/
$100 \mathrm{mmHg}$. There were no cervical bruits. Blood count, cerebrospinal fluid, radiographs of skull and chest, audiograms and caloric responses were normal. The ECG showed a sinus rhythm at $60 / \mathrm{min}$, a P-R interval of $0.16 \mathrm{sec}$, QRS of $0.08 \mathrm{sec}$ and normal complexes.

A diagnosis of transient vertebro-basilar ischaemia associated with attacks of paroxysmal tachycardia was made.

Case No. 609

A right-handed woman of 59 y'ears suddenly lost vision to her left for a period of $15 \mathrm{~min}$ in July 1962. In March 1963 she had an episode of dysphasia lasting about $2 \mathrm{hr}$. Finally, in April 1963 she suddenly developed pins and needles in the left upper limb and face and was again unable to see to her left ; these symptoms cleared after some minutes. She had, therefore, experienced first a right, then a left, and finally another right cerebral hemisphere disturbance. Examination revealed no abnormal neurological or cardiovascular signs. Blood pressure was $130 /$ $80 \mathrm{mmHg}$; pulse $76 / \mathrm{min}$ and regular. ECG was normal with a $P-R$ interval of $0 \cdot 14 \mathrm{sec}$ and $a$ QRS of $0.08 \mathrm{sec}$.

Whilst under observation she had two episodes of atrial fibrillation both of which subsided spontaneously and over the succeeding 4 years she continued to have occasional attacks of atrial fibrillation the cause of which was never found.

\section{TIAs and mitral stenosis}

TIAs may occur in association with rheumatic heart disease without atrial fibrillation (Hutchinson \& Stock, 1963). The following case is an example of this.

\section{Case No. 787}

A woman of 45 years developed at $18.30 \mathrm{hr}$ a right hemiparesis and dysphasia which cleared after $1 \mathrm{hr}$. She gave no history of cardiac irregularity at the time of the attack. When seen 5 days later there were no abnormal neurological signs, but she had a previously undiagnosed mitral stenosis and incompetence with regular rhythm. She was referred to a cardiologist who 
did not advise surgery. Anticoagulant therapy was commenced and she has remained well over 4 years.

It is often impossible to decide whether an episode of this kind is embolic or due to transient change in cardiac output. Emboli are unusual in the absence of atrial fibrillation, but an attack of fibrillation could have passed unnoticed by the patient. Equally, an attack of fibrillation could have been responsible for a transient fall in cardiac output, the focal character of the cerebral manifestations being determined by the site of pre-existing lesions in the cerebral arteries. Because of her age, the embolic hypothesis was preferred in the present instance and treatment instituted accordingly.

\section{TIAs associated with heart block}

Heart block commonly produces StokesAdams attacks, but when there is associated cerebrovascular disease, transient focal ischaemic attacks may also occur as in the following case.

Case No. 757

A man of 71 years began to experience in Spring 1963 sudden attacks of loss of consciousness without convulsion, lasting $20 \mathrm{~min}$, and occurring when he was sitting at a table halfway through a meal. He had ten attacks in 8 weeks, always during a meal, then remained free for 6 months; four more attacks, which were not associated with the taking of food, then followed. He next noticed that if he turned his head to left or right, his vision became 'grey' and if he persisted in the movement, he lost consciousness. He also had occasional, brief episodes of ataxia, seemingly unassociated with neck movement.

His pulse was found to be $36 / \mathrm{min}$ and regular and did not increase on gentle exercise. There was a systolic bruit over the left carotid artery and neck movement was restricted in all directions. On turning his head to the left he complained of greying of vision so the neck was returned to the neutral position. There were no abnormal neurological signs. An electrocardiogram confirmed that he had a complete heart block. He had further attacks of loss of consciousness whilst under observation in which the pulse was counted at $25 / \mathrm{min}$. He was transferred to the National Heart Hospital, where a pace-maker was successfully inserted. He had no further attacks over the next 4 years.

\section{TIAs and anaemia}

Severe anaemia is a well recognized cause of
TIAs (Siekert, Whisnant \& Millikan, 1960) which is illustrated by the following case.

Case No. 699

A man of 63 years suddenly developed vertigo, ataxia and diplopia with vertical separation of the images. This recurred intermittently throughout 1 day, being associated for part of the time with tingling across his neck and down both arms. One week later he had an episode of slurred speech; his tongue felt stiff and he had difficulty swallowing. His wife noted the left side of his face was drooping. The symptoms cleared after $6 \mathrm{hr}$.

Examination 6 weeks after the onset revealed only an extensor plantar response on the left. The blood pressure was $160 / 105 \mathrm{mmHg}$ in the right arm and $140 / 95 \mathrm{mmHg}$ in the left arm. The heart was normal and there were no vascular bruits. His haemoglobin was $5.4 \mathrm{~g} / 100 \mathrm{ml}$, MCV $68 \mu^{3}$ and MCHC $22 \%$. There were $1.1 \%$ reticulocytes. Tests for occult blood in the stools were positive on two occasions, but a barium meal and follow-through and a barium enema were normal. He received a transfusion of 4 pints of blood which brought his haemoglobin up to $9 \cdot 3 \mathrm{~g} / 100 \mathrm{ml}$. He had no further neurological episodes. He was re-admitted 8 weeks later witlo a history of increasing exertional dyspnoea of few weeks duration and evidence of cardia failure. His haemoglobin had fallen to $8.2 \mathrm{~g}$ $100 \mathrm{ml}$ and after further transfusion rose to $10 \cdot 2 \mathrm{~g}$. Serum iron was $105 \mu \mathrm{g} / 100 \mathrm{ml}$; the occult blood was still positive.

He was referred to a general medical unit where a further barium meal showed a small hiatus hernia, but neither barium swallow nor oesophagoscopy revealed any varices. Prolonged treatment with oral iron brought his haemoglobin up to $12.3 \mathrm{~g} / 100 \mathrm{ml}$ with a MCHC of $32 \%$ and occult blood gradually disappeared from his stools. He had no further TIAs.

This patient had experienced two TIAs in the vertebro-basilar territory in association with a severe anaemia. The latter was presumably due to gastro-intestinal bleeding, the source of which was never found.

\section{TIAs and polycythaemia}

Polycythaemia may also give rise to TIAs (Millikan, Siekert \& Whisnant, 1960) which respond to the treatment of the polycythaemia.

Case No. 512

A woman of 70 years began in November 1960 to have attacks of loss of vision in the left eye each lasting $5 \mathrm{~min}$; the attacks recurred every 2-3 weeks. In Febraury 1961 she also 
developed episodes of numbness and weakness of the right upper limb of $10 \mathrm{~min}$ duration. Investigation at that time showed her to have a blood pressure which varied from $195 / 100$ to $220 / 130 \mathrm{mmHg}$, a haemoglobin of $16.1 \mathrm{~g} / 100 \mathrm{ml}$ and a left carotid stenosis. She was treated with hypotensive and anticoagulant drugs without any clear-cut reduction in the frequency of her ischaemic episodes. In November 1963 she developed a left hemiparesis and re-investigation showed her haemoglobin to be $18 \mathrm{~g}$ with 6.5 million erythrocytes $/ \mathrm{mm}^{3}, 18,000$ leucocytes, and a haematocrit of $63 \%$. Further investigation (Dr C. S. Pitcher) revealed a blood volume of 5.9 litres $(100 \mathrm{ml} / \mathrm{kg})$, plasma volume $43 \mathrm{ml} / \mathrm{kg}$, red cell mass $56.6 \mathrm{ml} / \mathrm{kg}$. She was treated with radioactive phosphorus (Dr P. Wade) with considerable reduction in the frequency of her attacks. She required further treatment in September 1964 but since then has kept well with only occasional TIAs.

The patient had a carotid stenosis, hypertension, hypotensive therapy and polycythaemia, all of which could have contributed to her TIAs. The response to the treatment showed that the polycythaemia was an important factor.

\section{TIAs and leukaemia}

TIAs may be the presenting symptom in leukaemia as the next case shows.

Case No. 755

A man of 67 suddenly developed vertigo, ataxia and vomiting for $\frac{1}{2} \mathrm{hr}$ without tinnitus, deafness or other symptoms. Three weeks later he had a second attack lasting several hours. Over the preceding 7 months he had lost 1 stone in weight and had several night sweats.

Examination showed venous engorgement of the retinae with extensive haemorrhages containing white centres along the veins. Some of the haemorrhages were old, some new; there was no papilloedema. The liver and spleen were enlarged, but there was no lymphadenopathy. In the nervous system there was slight Rombergism and ataxia of gait.

Blood examination showed a haemoglobin of $5.8 \mathrm{~g} / 100 \mathrm{ml}$ with 700,000 white cells $/ \mathrm{mm}^{3}, 27 \%$ of which were lymphocytes. A diagnosis of lymphatic leukaemia was made.

This patient was also severely anaemic, but TIAs may occur in patients with leukaemia who are not anaemic.

\section{'Little strokes' and thrombotic thrombocytopenia purpura}

A rare blood disorder which may present with cerebrovascular episodes is thrombotic thrombo- cytopenia purpura of which the next case is an example.

Case No. 611

A woman of 57 years was found speaking incoherently and unable to use her right arm. These symptoms were improving when she developed a raised, red rash all over the body. This faded, but 2 weeks later she suddenly developed a left hemiplegia, hemianopia and neglect of the left side of her body. She was admitted to hospital on 21 June 1963 under the care of Dr Q. J. G. Hobson and after $24 \mathrm{hr}$ she developed extensive bruising. Her haemoglobin was $10.8 \mathrm{~g} / 100 \mathrm{ml}$, white cells $5800 / \mathrm{mm}^{3}$, platelets $92,000 / \mathrm{mm}^{3}$, reticulocytes $8 \cdot 4 \%$. Bonemarrow examination revealed hyperactive normoblastic erythropoiesis and a normal granulocyte series. The megakaryocytes numbered $0.5 \%$ of the differential count, and there was little evidence of platelet formation. The Quick onestage prothrombin time was $20 \mathrm{sec}$ (control $19 \mathrm{sec}$ ) coagulation time (Dale and Laidlaw) 2 min. Direct Coombs' test was negative with serum dilutions of $1: 4$ to $1: 1024$. Serum bilirubin $1.4 \mathrm{mg} / 100 \mathrm{ml}$. Serum protein $6.0 \mathrm{~g} / 100 \mathrm{ml}$ with normal electrophoretic strip.

Over the course of 10 days her platelets fell to $27,000 / \mathrm{mm}^{3}$, thrombotic thrombocytopenia purpura was diagnosed and heparin 10,000 units intravenously 4-hourly was given. She improved on this regime, the platelet count rising to $209,000 / \mathrm{mm}^{3}$, though her hemiplegia remained unchanged. She was able to return home on 14 September but was re-admitted on 8 November, having developed a brain-stem lesion with dysarthria, dysphagia and ataxia. Her haemoglobin was $13.1 \mathrm{~g}$ and her platelet count $121,000 / \mathrm{mm}^{3}$. Her condition slowly deteriorated and she died on 28 February 1964.

Necropsy (Dr A Knudsen) showed areas of haemorrhage into the muscle of the anterior wall of both cardiac ventricles, and on the surface of the kidneys. There were some renal infarcts measuring up to $2 \mathrm{~cm}$ diameter. The spleen was firm, but the follicles were ill-defined. The brain showed multiple areas of cortical softening, measuring up to $3 \mathrm{~cm}$ in diameter. The white matter was not involved. The spinal cord was normal. Sections showed plugging of capillary vessels by hyaline and granular eosinophilic material, best seen in the kidney, heart, pancreas and bladder.

\section{'Little strokes' associated with hypertension}

Hypotension is a well-recognized cause of TIAs (Denny-Brown \& Meyer, 1957), but their 
occurrence in association with hypertension is less well recognized. Indeed, when hypertension is being treated with hypotensive drugs, the mistake of attributing TIAs to hypotension rather than hypertension may be made. This dilemma is illustrated by the next case.

Case No. 810

A woman of 64 years awoke one morning in December 1964 with headache, vomiting, weakness of the right leg and unsteadiness of gait which was greater than could be accounted for by her weakness. The vomiting and weakness ceased after a few hours, but she continued to be unsteady for about 2 weeks. She was seen at a hospital where her blood pressure was found to be raised and treatment with hypotensive drugs begun.

Over the next 3 months she had repeated attacks of non-rotatory 'giddiness'. As there was doubt as to whether this symptom was due to hypotension produced by therapy or hypertension she was referred for a further opinion. While in the supine position on the examination couch she suddenly complained of feeling faint and giddy. A second-degree nystagmus to the left appeared, accompanied by a left facial weakness of nuclear type, ataxia in the left upper limb and increase of reflexes down the right side. She vomited several times.

Unfortunately the examination had not reached the stage at which the blood pressure was recorded before the onset of these symptoms, but a few minutes after the onset it was 260/ $150 \mathrm{mmHg}$. Over the next few hours it fell to $220 / 130 \mathrm{mmHg}$.

Treatment of her blood pressure with methyldopa was intensified and a level of $160 / 110 \mathrm{mmHg}$ maintained. The nystagmus and ataxia disappeared over the course of 2 weeks, but there was persistent increase in the reflexes on the right.

Five weeks later she had a further attack of giddiness with a re-appearance of nystagmus; her blood pressure had been rising slowly over the previous $48 \mathrm{hr}$, reaching a level of $220 /$ $140 \mathrm{mmHg}$ at the time of the attack; control was again established at a level of $170-150 / 110$ $100 \mathrm{mmHg}$ and she had no further symptoms.

\section{'Little strokes' with multiple causes}

The difficulty in determining which of several possible causes is responsible for cerebrovascular episodes was mentioned in the report of the patient with polycythaemia (Case No. 512) and is further illustrated by the next case in which hypertension and blood loss were both present.
Case No. 808

A man of 58 years developed melaena in October 1964. His haemoglobin was reduced to $\stackrel{\varnothing}{\circledR}$ $4.9 \mathrm{~g}$ and a transfusion of 5 pints of blood was $c$ given. He had no neurological symptoms at that $\vec{F}$ time. A barium meal showed scarring of the duodenal cap.

He remained well until March 1965 when both $\frac{\overline{ }}{\bar{n}}$ lower limbs suddenly became weak as he stepped $\frac{0}{\vec{D}}$ out of his car. He was helped into the house $\stackrel{\varnothing}{\unrhd}$ where he observed that the left arm was weak $\%$ and that there was a sensation of pins and $\overrightarrow{0}$ needles in the left lower limb. The symptoms ? began to improve within 3 days of the onset but $\vec{\omega}$ 7 days later he had a severe melaena causing syncope and necessitating a further transfusion $\frac{0}{3}$ of 6 pints of blood. This was not accompanied by an exacerbation of his neurological symptoms.

Examination 5 weeks later showed only slight weakness in the left upper limb with more severe weakness in the left lower limb; the tendon reflexes were brisker on the left and the plantar was extensor. Blood pressure was $180 / 110 \mathrm{mmHg}$. There was a late systolic murmur at the apex, the retinae showed only grade I changes, there were no vascular bruits but the ECG showed evidence of left ventricular hypertrophy. A\& arch aortogram showed no abnormality of the carotid and vertebral arteries.

The problem as to whether his neurological symptoms were assocated with his blood loss or with his hypertension was discussed. The former was thought to be unlikely because there was no exacerbation of his neurological deficit at the time of the second melaena which was severe enough to produce syncope. Accordingly his blood pressure was reduced with methyldopa $250 \mathrm{mg}$ t.d.s. and over a period of $2 \frac{1}{2}$ years he has remained well with a blood pressure of $150 / 100 \mathrm{mmHg}$.

Acute blood loss poses a special problem because it may be associated with hypotension, so that it may be difficult to determine whether the anaemia or the fall in blood pressure is the cause of the cerebrovascular symptoms. The time-relationships in the present case indicate that probably neither was responsible, but the difficulty in apportioning responsibility among several causes and so deciding treatment, should not be underestimated.

'Little strokes' associated with arterial constriction

Not every arterial stenosis is due to an atheromatous narrowing; a healthy vessel may be constricted from without and give rise to symptoms. 
Case No. 928

A man of 64 years woke on the morning of the 25th January 1967 with weakness of the left arm and leg. At noon the following day the right side began to tingle and became weak. He lost consciousness for a few moments and on recovery was found to be dysarthric. Examination at this stage revealed no other abnormality in the cranial nerves. All four limbs were weak with increased reflexes and extensor plantar responses. Position sense was impaired in the left lower limb. By the 29 January power had returned except in the left leg which dragged as he walked. Blood pressure was $150 / 95 \mathrm{mmHg}$ in both arms and neck movements were full. Arch aortography showed severe constriction of the left vertebral artery at the level of C6-7 space. The right vertebral artery was small in calibre, the carotids were normal.

At operation (Professor G. W. Taylor) the left vertebral artery was found to be constricted by fibro-muscular bands at the C6-7 level just before it entered the bony canal in the transverse process of .C6. The vessel itself appeared healthy. The bands were divided to free the artery. Postoperative angiography showed the constriction to have been relieved; the patient has had no further TIAs.

\section{'Little strokes' and meningo-vascular syphilis}

A time-honoured cause of stroke which nowadays tends to be overlooked is neurosyphilis. The following case underlines the need to examine the cerebrospinal fluid in every case of stroke.

\section{Case No. 720}

A man of 56 years woke from an afternoon nap to find his left arm and leg to be paralysed. On admission to hospital he had a left hemiplegia involving the face, arm and leg and a blood pressure of $200 / 90 \mathrm{mmHg}$. The pupillary reflexes were normal. The cerebrospinal fluid contained ninety-five lymphocytes and $64 \mathrm{mg}$ of protein $/ 100 \mathrm{ml}$. The WR was positive in the blood and cerebrospinal fluid. He made an almost complete recovery following treatment with 1.2 mega-units of procaine penicillin for 21 days.

\section{'Little strokes' and cerebral tumours}

It is not only vascular lesions which may present as TIAs; cerebral tumours may first be manifest in this way.

Case No. 933

A man of 54 years awoke in September 1965 with numbness of the left upper limb and clum- siness in the performance of tasks such as the fastening of buttons. This improved over 4 days. When seen in October, neurological examination was negative. Radiographs of the skull showed the calcified pineal to be central. The electroencephalogram showed diffuse irregular slow activity in both hemispheres with one episode of focal sharp waves, which could have been artefact, in the right frontal region. A second examination 1 month later showed diffuse slow activity as before, but no right frontal disturbance.

In July 1966, a second episode occurred, but again improved. He was admitted to hospital in September 1966 when examination revealed nothing abnormal except a threshold of $1 \mathrm{~cm}$ to twopoint discrimination in the left hand. Archaortography showed no lesion of the carotid and vertebral arteries in their cervical course. Electroencephalography showed diffuse activity at 4-5 cycles/sec with sporadic delta activity in the right mid-temporal region on overbreathing. Following discharge he returned to work, but began to have morning headache, vomited occasionally and had difficulty using his left arm and leg. He was re-admitted on 14 October 1966 when a right carotid angiogram revealed a right parietal neoplasm which, at operation (Mr Lindsay Symon), proved to be a meningioma. The patient made an excellent recovery.

Case No. 789

A man of 61 years gave a 3-year history of episodes of headaches and vomiting lasting $24 \mathrm{hr}$ on three or four occasions each year. Three months prior to being seen he began to have occasional, brief attacks of numbness of the right hand. Sixteen days before being seen he suddenly developed numbness of the right index and middle finger; this disturbance spread within seconds to include the whole of the right upper limb and right side of face and trunk, but not the lower limb. The symptom persisted for $10 \mathrm{~min}$ during which time the right arm hung limply by his side and he was unable to speak properly. These attacks recurred frequently but between them he had no disability. He was started on heparin, whereupon the attacks ceased for $48 \mathrm{hr}$. Warfarin had been started with the heparin, hence the latter was stopped after $48 \mathrm{hr}$, whereupon the patient had first a short-lived attack and then a more prolonged attack lasting some hours. A continuous intravenous infusion of heparin was begun and he had no further attacks over $48 \mathrm{hr}$. The heparin was again stopped prior to angiography and he had three slight attacks. The angiogram showed a left parietal neoplasm 
which at biopsy proved to be a grade II astrocytoma.

Not only was the description of the attacks typical of TIAs but they also showed a striking arrest following the administration of heparin on two occasions with recurrence when the heparin was stopped.

\section{Discussion}

Transient disturbances of neurological function may be associated with a number of conditions. They may for instance occur in epilepsy as in Todd's paralysis, though usually the history of a preceding convulsion makes clear the nature of the transient weakness. Similarly, the reason for the temporary paresis which may follow focal motor seizures is revealed by the account of clonic movements of the affected part. Focal sensory attacks, however, present more of a problem; it is often extremely difficult to determine whether transient sensory symptoms are a positive phenomenon due to an epileptic discharge from the sensory cortex, or a negative phenomenon due to ischaemic paralysis of that area. The duration of the disturbance may help as it is unusual for an epileptic discharge to last more than 1 or $2 \mathrm{~min}$; a numbness lasting $10 \mathrm{~min}$ or so is, therefore, more likely to be ischaemic.

The motor, sensory and visual symptoms which may occur in association with migraine may also pose problems. The symptoms which may usher in an attack may be very like TIAs especially in those cases in which the succeeding headache is mild. Indeed, it may be argued that these are in effect TIAs, as it is commonly held that the symptoms at the onset of an attack of migraine are due to constriction of intracranial arteries. Further difficulty is created by the fact that TIAs associated with atheroma may be accompanied, or followed, by localized headache due to dilatation of collateral vessels in an attempt to ward off the threatened ischaemia.

Even when it seems clear that a transient focal cerebral disturbance is due to ischaemia, the possibility that it is the result of a pathology other than atherosclerosis must be borne in mind, as has been abundantly illustrated by the case histories presented here. A careful history and examination should reveal most of these. In addition, simple investigations such as blood count, ECG and radiographs of the cervical spine should bring to light most of the remainder.

It is the cerebral tumour presenting with TIAs which offers the most difficulty. Echoencephalography and electroencephalography are of great help in this situation. The finding of a focal, slow wave disturbance in the EEG in a patient currently experiencing TIAs should always ڤ arouse suspicion of a tumour as transient focal $\frac{\vec{\phi}}{\mathrm{d}}$ ischaemia, unlike an infarct, does not produce a $\unrhd$ disturbance of this kind. If the focal disturbance $\stackrel{C}{-}$ persists over 3 weeks, it should certainly be $\underset{\rightarrow}{\vec{F}}$ regarded as due to a tumour until proved otherwise. Cerebral angiography or a radioactive brain scan must be undertaken.

Even when it is decided to carry out angiography, the difficulties are not at an end. The study of patients experiencing TIAs has placed $\omega$ increasing emphasis upon the great vessels in the $\overrightarrow{0}$ neck and their origins from the aortic arch, as the site of the relevant lesions; these are best $\vec{\omega}$ demonstrated by aortography via a catheter introduced into the arch of the aorta by one or other route. This procedure does not, however, permit adequate visualization of the intracranial vessels, for which selective puncture or $\vec{\omega}$ catheterization of single vessels with radiographs $i$ centred on the skull is preferable. There is a $\vec{\omega}$ conflict of interests here which cannot be resolved 0 by any simple rule-of-thumb; the most appropriate procedure must be determined for each $\subsetneq$ case in the light of the clinical features and the results of other investigations. When there is sus- $\vec{\varphi}$ picion of a cerebral neoplasm, selective angiog graphy of a single artery is the better procedure But when cervical bruits or other evidence make atheroma the more likely cause of TIA $\overrightarrow{s,}$ aortography is more informative, as it may assess the status of all four vessels supplying the brain.

In all these circumstances the most important factor remains the clinician's awareness of the possibilities. Because atherosclerotic vascular disease is so common, there is always the danger of a false sense of diagnostic certainty arising. These cases illustrated here indicate how misplaced this may be.

\section{Acknowledgment}

I wish to thank the British Heart Foundation for financial support of my studies in cerebrovascular disease, of which this forms a part.

\section{References}

DenNy-Brown, D. \& Meyer, J.S. (1957) The cerebral $N$ collateral circulation. II. Production of cerebral infarction $\sigma$ by ischemic anoxia and its reversibility in early stages. $N$ Neurology (Minneap.), 7, 567.

Hutchinson, E.C. \& STock, J.P.P. (1963) Paroxysmal $\omega$ cerebral ischaemia in rheumatic heart-disease. Lancet, ii, $\underset{<}{\sigma}$ 653.

Millikan, C.H., Siekert, R.G. \& Whisnant, J.P. (1960) Intermittent carotid and vertebral basilar insufficiency $\mathbb{D}$ associated with polycythaemia. Neurology (Minneap.), 10, 188.

Siekert, R.G., Whisnant, J.P. \& Millikan, C.H. (1960) Anaemia and intermittent focal cerebral arterial insufficiency. Arch. Neurol. (Chic.), 3, 386. 